\title{
ON SEMICONNECTED MAPPINGS OF TOPOLOGICAL SPACES
}

\author{
JOHN JONES, JR.
}

1. Introduction. Let $(X, \mathfrak{u})$ and $(Y, \mathcal{V})$ denote topological spaces as in Kelly [1]. A mapping $f$ of $(X, \mathcal{U})$ into $(Y, \mathcal{V})$ is said to be connected if and only if it maps connected subsets of $(X, \mathfrak{u})$ into connected subsets of $(Y, \vartheta)$. W. J. Pervin and N. Levine [3] and T. Tanaka [4] recently considered connected mappings of Hausdorff spaces $(X, \mathfrak{u})$ into $(Y, \mathcal{V})$. A mapping $f$ of $(X, \mathfrak{U})$ into $(Y, \mathcal{V})$ is semiconnected if $f^{-1}(A)$ is a closed and connected set in $(X, \mathcal{U})$ whenever $A$ is a closed and connected set in $(Y, \mathcal{V})$. A mapping $f$ is bi-semiconnected if and only if $f$ and $f^{-1}$ are each semiconnected. Using the definition of G. T. Whyburn [5] a connected $T_{1}$-space $(X, \mathcal{U})$ is said to be semilocally connected (s.l.c.) at $x \in X$ if and only if there exists a local open base at $x \in X$ such that $X \backslash V$ has only a finite number of components, where $V$ is any element of the local open base at $x$.

Since continuous mappings are special cases of connected mappings it is of interest to know what conditions must be placed upon a given mapping or upon the topological spaces $(X, \mathfrak{u}),(Y, \mathcal{V})$ in order to conclude that a given mapping $f$ is continuous or is a homeomorphism. Examples of connected mappings which are not continuous are given by C. Kuratowski [2] and Pervin and Levine [3].

\section{Results.}

TheOREM 1. Let $f$ be a one-to-one onto semiconnected mapping of a topological space $(X, \mathcal{u})$ to a semilocally-connected topological $T_{2}$ space $(Y, V)$, then $f$ is continuous.

Let $B$ be an open set in $Y$, and $f^{-1}(B)=A \subseteq X$. Choose a point $x \in A$ and let $f(x)=y \in B$. Since $(Y, \mathcal{V})$ is semilocally-connected there exists an open set $B_{y} \subseteq B$ containing $y$ and $Y \backslash B_{y}$ consists of a finite number of distinct components. Let these components be designated by $B_{1}, B_{2}, B_{3}, \cdots, B_{n}$. Then $B_{y}=Y \backslash \bigcup_{i=1}^{n} B_{i}$, where each $B_{i}$ is connected and closed. Let $A_{i}=f^{-1}\left(B_{i}\right)$, for $i=1,2, \cdots, n$. Each $A_{i}$ is closed and connected since $f$ is semiconnected. Now either $x$ belongs to the closure of some $A_{j}$ or it does not. Suppose that $x$ belongs to the closure of some $A_{j}$, for some $j=1,2, \cdots, n$. Now $A_{j} \cup_{x}$ is closed and connected since $f$ was a semiconnected mapping. Thus $f\left(A_{j} \cup x\right)=B_{j}$

Received by the editors October 20, 1966 and, in revised form, December 15, 1966. 
which is connected. So $y \in B_{j}$, but this is impossible since $B_{j}$ is a component of $Y \backslash B_{y}$ which does not contain $y$. So $x$ cannot belong to the closure of $A_{j}$ for any $j=1,2,3, \cdots, n$.

There exists an open set $O_{i}=X \backslash \mathrm{cl} A_{i}$ such that $x \in O_{i}, i=1,2,3$, $\cdots, n$. Now $O_{x}=\bigcap_{i=1}^{n} O_{i}$ is an open set in $X$ containing $x$. Also $f\left(A_{i}\right)=B_{i}$, and $f\left(\mathrm{cl} A_{i}\right) \supseteq B_{i}$. So we have $Y \backslash f\left(\mathrm{cl} A_{i}\right) \subseteq Y \backslash B_{i}$. Also

$$
\begin{aligned}
f\left(O_{x}\right) & =f\left(\bigcap_{i=1}^{n} O_{i}\right)=\bigcap_{i=1}^{n} f\left(O_{i}\right)=\bigcap_{i=1}^{n} f\left(X \backslash \mathrm{cl} A_{i}\right) \\
& =\bigcap_{i=1}^{n}\left[Y \backslash f\left(\operatorname{cl} A_{i}\right)\right] \subseteq \bigcap_{i=1}^{n}\left[Y \backslash B_{i}\right]=Y \backslash \bigcap_{i=1}^{n} B_{i}=B_{\nu} .
\end{aligned}
$$

Since $f$ is a one-to-one onto mapping and for any $B_{y}$ there exists an $O_{x}$ such that $f\left(O_{x}\right) \subseteq B_{y}, f$ is a continuous mapping of $(X, \mathcal{u})$ into $(Y, \mathcal{V})$.

Theorem 2. Let $(X, \mathcal{u}),(Y, \mathcal{\cup})$ be semilocally-connected and $\wedge T_{2}$ topological spaces and $f$ be a one-to-one bi-semiconnected mapping of $(X$, $u)$ onto $(Y, \mathcal{V})$, then $f$ is a homeomorphism.

According to Theorem 1 above $f$ is continuous, and applying the same type argument as used in the proof of Theorem 1, we see that $f^{-1}$ is a one-to-one onto continuous mapping of $(Y, \mathcal{V})$ to $(X, \mathcal{u})$. Hence $f$ is a homeomorphism.

The author wishes to acknowledge the helpful comments of the referee.

\section{REFERENCES}

1. J. L. Kelly, General topology, Van Nostrand, New York, 1955.

2. C. Kuratowski, Topologie. II, Hafner, Warsaw, 1952.

3. W. J. Pervin and N. Levine, Connected mappings of Hausdorff spaces, Proc. Amer. Math. Soc. 11 (1958), 488-496.

4. T. Tanaka, On the family of connected subsets and the topology of space, J. Math. Soc. Japan 7 (1955), 389-393.

5. G. T. Whyburn, Analytic topology, Amer. Math. Soc. Colloq. Publ., Vol. 28, Amer. Math Soc., Providence, R. I., 1942.

George Washington University 